\title{
Melioidosis: Deadly bacteria in Gazipur soil of Bangladesh
}

Scientists of Ibrahim Medical College and Dhaka University have recently found a fatal infectious bacterium in the soil of Kapasia in Gazipur, for the first time in Bangladesh. Known as Burkholderia pseudomallei, these bacteria can cause melioidosis, a rare disease that leads to serious infections of lung, blood and other organs.

Melioidosis, caused by the gram-negative saprophyte Burkholderia pseudomallei, is a disease of public health importance in Southeast Asia and northern Australia that is associated with high case-fatality rates in animals and humans. It has the potential for epidemic spread to areas where it is not endemic, and sporadic case reports elsewhere in the world suggest that as-yet-unrecognized foci of infection may exist. The bacteria can invade human body through skin, inhalation and contaminated food.

Melioidosis symptoms most commonly stem from lung disease where the infection can form a cavity of pus (abscess). The effects can range from mild bronchitis to severe pneumonia. As a result, patients also may experience fever, headache, loss of appetite, cough, chest pain, and general muscle soreness. The effects can also be localized to infection on the skin (cellulitis) with associated fever and muscle aches. It can spread from the skin through the blood to become a chronic form of melioidosis affecting the heart, brain, liver, kidneys, joints, and eyes.

Environmental determinants of this infection, apart from a close association with rainfall, are yet to be elucidated. Disease manifestations are protean, and no inexpensive, practical, and accurate rapid diagnostic tests are commercially available; diagnosis relies on culture of the organism. Despite the introduction of ceftazidime- and carbapenem-based intravenous treatments, melioidosis is still associated with a significant mortality attributable to severe sepsis and its complications. A long course of oral eradication therapy is required to prevent relapse. Studies exploring the role of preventative measures, earlier clinical identification, and better management of severe sepsis are required to reduce the burden of this disease.

Since 2001, doctors of Ibrahim Medical College have detected five such patients, who are also diabetic. All these patients came from Mymensingh-Gazipur region, which led scientists to look for the bacteria in the area. If melioidosis remains undiagnosed and untreated for long, chances of death are 80 percent.

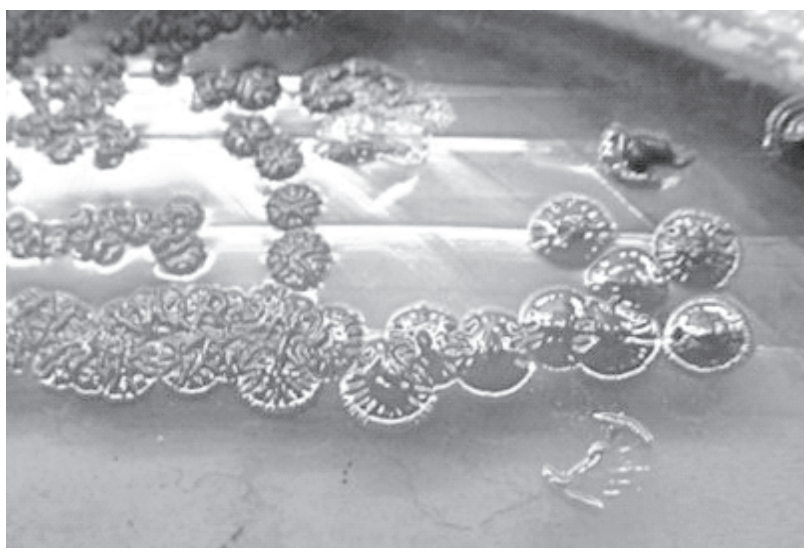

Figure: Photomicrograph of Burkholderia pseudomallei Microbiology Lab, Ibrahim Medical College

Similar to tuberculosis in many ways, the disease usually requires four to six months of antibiotic treatment that can become quite expensive when the germ turns out to be resistant to the common antibiotics. Scientists and doctors say the number of people infected by the bacteria might be higher, but the germ goes undetected because of the ignorance of doctors and microbiologists about the presence of these bacteria. This germ may silently live in a human body for decades. It manifests its presence through various symptoms when the carrier person's immune system is damaged by diabetes, kidney failure, liver cirrhosis and AIDS. "We have had patients who were infected by the bacteria. But we could not detect its origin. Now we have found the bacteria in the soil of Kapasia in Gazipur," said J Ashraful Haq, a professor of microbiology at Ibrahim Medical College. Statistics on how many people in Bangladesh are infected by the bacteria are not available, said the professor, who has been conducting a research to detect the germ for more than a decade. He first detected the bacteria in a diabetic patient in 2001. In the next few years he detected several other cases of melioidosis in diabetic patients - all coming from Mymensingh and Tangail. This led him and Shariful Alam Jilani, another professor of the same department, and Prof Chowdhury Rafiqul Ahsan and Jamshedul Alam of Dhaka University to start a research to ascertain the source of the bacteria in the beginning of last year. They finally found it in the soil of Kapasia late last month. They are looking for the germ in the soil of other parts of the country.

Available from: http://www.thedailystar.net/beta2/ news/ deadly-bacteria-in-gazipur-soill 